\title{
Human Face Recognition with Different Statistical Features
}

\author{
Javad Haddadnia ${ }^{1}$, Majid Ahmadi ${ }^{1}$, and Karim Faez ${ }^{2}$ \\ ${ }^{1}$ Electrical and Computer Engineering Department, University of Windsor \\ Windsor, Ontario, Canada, N9B 3P4 \\ \{javad, ahmadi\}@uwindsor.ca \\ ${ }^{2}$ Electrical Engineering Department, Amirkabir University of Technology \\ Tehran, Iran, 15914 \\ kfaez@cic.aku.ac.ir
}

\begin{abstract}
This paper examines application of various feature domains for recognition of human face images to introduce an efficient feature extraction method. The proposed feature extraction method comprised of two steps. In the first step, a human face localization technique with defining a new parameter to eliminate the effect of irrelevant data is applied to the facial images. In the next step three different feature domains are applied to localized faces to generate the feature vector. These include Pseudo Zernike Moments (PZM), Principle Component Analysis (PCA) and Discrete Cosine Transform (DCT). We have compared the effectiveness of each of the above feature domains through the proposed feature extraction for human face recognition. The Radial Basis Function (RBF) neural network has been utilized as classifier. Simulation results on the ORL database indicate the effectiveness of the proposed feature extraction with the PZM for human face recognition.
\end{abstract}

\section{Introduction}

In recent years there has been a growing interest in machine recognition of faces due to potential commercial application such as film processing, law enforcement, person identification, access control systems, etc. A recent survey of the face recognition systems can be found in reference [1]. The ultimate goal of designing human face recognition systems is to develop different feature extractions and classification schemes that achieve the best possible recognition performance.

A complete conventional human face recognition system should include three stages. The first stage involves detecting the location of face in arbitrary images. Although many researchers tried to solve this problem [2-3], however, detecting the location of a face is still difficult and complicated due to unknown position, orientation and scaling of face in an arbitrary image. The second stage requires extraction of pertinent features from the face image. Two main approaches to feature extraction have been extensively used by other researchers [4]. The first one is based on extract- 
ing structural and geometrical facial features that are local structure of face images, for example, the shapes of the eyes, nose and mouth. The structural-based approaches deal with local data instead of global data. It has been shown that structural-based approaches by explicit modeling of facial features have been troubled by the unpredictability of face appearance and environmental conditions. The second method is statistical-based approaches that extract features from the whole image and therefore use global data instead of local data. Since the global data of an image are used to determine the feature elements, data that are irrelevant to facial portion such as hair, shoulders and background may contribute to creation of erroneous feature vectors that can affect the recognition results [5]. Finally the third stage involves classification of facial images based on the derived feature vector. Neural networks have been employed and compared to conventional classifiers for a number of classification problems. The results have shown that the accuracy of the neural network approaches are equivalent to, or slightly better than, other methods. Also, due to the simplicity, generality and good learning ability of the neural networks, these types of classifiers are found to be more efficient [6].

In this paper a new feature extraction technique is developed. This algorithm is based on the face localization using the shape information [3] and a definition of a new parameter for eliminating the irrelevant data from arbitrary face images. This parameter is named Correct Information Ratio (CIR). We have shown how CIR can improve the recognition rate. Once the face localization process was completed, a subimage is created and then PZM, PCA and DCT are computed on the subimage to generate the feature vector associated with each image. These feature vectors are sent to classifier, which is RBF neural network. The recognition performance of each feature domain is subsequently analyzed and compared. The organization of this paper is as follows: Section 2 presents face localization and feature extractionmethods. In section 3, feature domains are presented. Classifier techniques are described in section 4 and finally, section 5 and 6 presents the experimental results and conclusions.

\section{Face Localization and Feature Extraction}

The ultimate goal of the face localization is finding an object in an image as a face candidate that its shape resembles the shape of a face. Many researchers have concluded that an ellipse generally can approximate the face of a human [2-4]. Considering the elliptical shape of a face in general, it is convenient to search for connected components using a region-growing algorithm and fit an ellipse to every connected component of nearly elliptical shape. A technique is presented in [3], which finds the best-fit ellipse to enclose the facial region of the human face in a frontal view of facial image.

The aim of the feature extractor is to produce a feature vector containing all pertinent information about the face to be recognized. The feature vector generation is very important in any high accuracy pattern recognition system. In this paper global data from a facial image are used to derive the feature vector. It is important that in this phase all irrelevant data pertaining to the face images such as hair, shoulders and background be eliminate and to keep only the important data about the face images. 
Our feature extraction method therefore has two different steps. In the first step a subimage is created to enclose only the important information about the face in an ellipse. In the second step, feature vector elements are determined by computing PZM, PCA and DCT on the derived subimage.

The subimage encloses all pertinent information around the face candidate in an ellipse while pixel values outside the ellipse are set to zero. Unfortunately through creation of the subimage with the best-fit ellipse many unwanted regions of the face image may still appear in this subimage, as shown in Fig. (1). These include hair portion, neck and part of the background. To overcome this problem, instead of using the bestfit ellipse for creating subimage we have defined another ellipse. The proposed ellipse has the same orientation and center as the best-fit ellipse but the length of the major (A ) and the minor (B) axis are calculated as follows:

$$
\mathrm{A}=\rho . \alpha \quad, \quad \mathrm{B}=\rho . \beta
$$

Where $\alpha$ and $\beta$ are the length of the major and minor axis of the best-fit ellipse [3] and the coefficient $\rho$ that we have named Correct Information Ratio (CIR) varies from 0 to 1. Fig. (2) shows the effect of changing CIR while Fig. (3) shows the corresponding subimages. Our exprimental results with 400 images show that the best value for CIR is around 0.87 . By using a subimage with CIR parameter, data that are irrelevant to facial portion are disregarded. Also the speed of computing feature domains is increased due to smaller nonzero pixels content of the subimages.
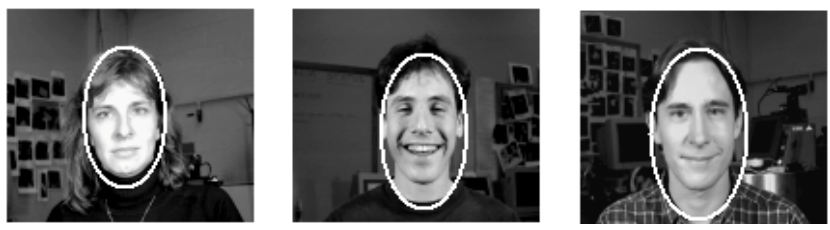

Fig. 1. Face localization method

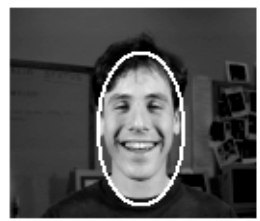

$\rho=1.0$

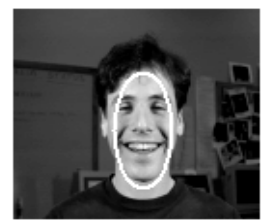

$\rho=0.7$

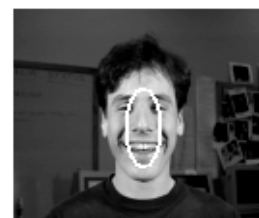

$\rho=0.4$

Fig. 2. Different ellipses with related CIR value
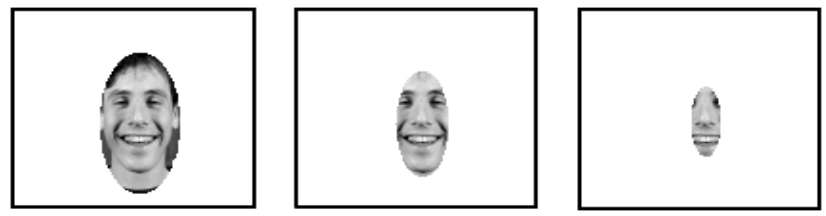

Fig. 3. Creating subimage based on CIR value 


\section{Feature Domains}

In order to design a good face recognition system, the choice of feature domains is very crucial. To design a system with low to moderate complexity the feature vectors should contain the most pertinent information about the face to be recognized. In this paper different feature domains are extracted from the derived subimages. These include PZM, PCA and DCT.

\subsection{Pseudo Zernike Moment (PZM)}

The advantages of considering orthogonal moments are that they are shift, rotation and scale invariant and very robust in the presence of noise. The PZM of order $n$ and repetition $\mathrm{m}$ can be computed using the scale invariant central moments and the radial geometric moments that defined in reference [8] as follows:

$$
\begin{aligned}
\mathrm{PZM}_{\mathrm{nm}} & =\frac{\mathrm{n}+1}{\pi} \sum_{(\mathrm{n}-\mathrm{m}-\mathrm{s}) \text { even, }, \mathrm{s}=0}^{\mathrm{n}-|\mathrm{m}|} \mathrm{D}_{\mathrm{n},|\mathrm{m}|, \mathrm{s}} \sum_{\mathrm{a}=0}^{\mathrm{k}} \sum_{\mathrm{b}=0}^{\mathrm{m}}\left(\begin{array}{l}
\mathrm{k} \\
\mathrm{a}
\end{array}\right)\left(\begin{array}{l}
\mathrm{m} \\
\mathrm{b}
\end{array}\right)(-\mathrm{j})^{\mathrm{b}} \mathrm{CM}_{2 \mathrm{k}+\mathrm{m}-2 \mathrm{a}-\mathrm{b}, 2 \mathrm{a}+\mathrm{b}} \\
& +\frac{\mathrm{n}+1}{\pi} \sum_{(\mathrm{n}-\mathrm{m}-\mathrm{s}) \text { odd }, \mathrm{s}=0}^{\mathrm{n}-|\mathrm{m}|} \mathrm{D}_{\mathrm{n},|\mathrm{m}|, \mathrm{s}} \sum_{\mathrm{a}=0}^{\mathrm{d}=0} \sum_{\mathrm{b}=0}^{\mathrm{m}}\left(\begin{array}{l}
\mathrm{d} \\
\mathrm{a}
\end{array}\right)\left(\begin{array}{l}
\mathrm{m} \\
\mathrm{b}
\end{array}\right)(-\mathrm{j})^{\mathrm{b}} \mathrm{RM}_{2 \mathrm{~d}+\mathrm{m}-2 \mathrm{a}-\mathrm{b}, 2 \mathrm{a}+\mathrm{b}}
\end{aligned}
$$

where $\mathrm{k}=(\mathrm{n}-\mathrm{s}-\mathrm{m}) / 2, \mathrm{~d}=(\mathrm{n}-\mathrm{s}-\mathrm{m}+1) / 2, \mathrm{CM}_{\mathrm{i}, \mathrm{j}}$ is the Central moments, $\mathrm{RM}_{\mathrm{i}, \mathrm{j}}$ is the Radial moments [8] and $\mathrm{D}_{\mathrm{n}, \mathrm{m} \mid \mathrm{s}}$ is defined as:

$$
\mathrm{D}_{\mathrm{n},|\mathrm{m}| \mathrm{s}}=(-1)^{\mathrm{s}} \frac{(2 \mathrm{n}+1-\mathrm{s}) !}{\mathrm{s} !(\mathrm{n}-|\mathrm{m}|-\mathrm{s}) !(\mathrm{n}-|\mathrm{m}|-\mathrm{s}+1) !}
$$

\subsection{Principle Component Analysis (PCA)}

PCA is a well-known statistical technique for feature extraction [9]. Each $\mathrm{M} \times \mathrm{N}$ image in the training set was row concatenated to form $M N \times 1$ vectors $x_{i}$. Given a set of $\mathrm{N}_{\mathrm{T}}$ training images $\left\{\mathrm{x}_{\mathrm{i}}\right\}_{\mathrm{i}=0,1, \ldots \mathrm{N}_{\mathrm{T}}}$ the mean vector of the training set was obtained as:

$$
\overline{\mathrm{x}}=\frac{1}{\mathrm{~N}_{\mathrm{T}}} \sum_{\mathrm{i}=1}^{\mathrm{N}_{\mathrm{T}}} \mathrm{x}_{\mathrm{i}}
$$

A $\mathrm{N}_{\mathrm{T}} \times \mathrm{MN}$ training set matrix $\mathrm{X}=\left[\mathrm{x}_{\mathrm{i}}-\overline{\mathrm{x}}\right]$ can be built. The basis vectors are obtained by solving the eigenvalue problem:

$$
\Lambda=\mathrm{V}^{\mathrm{T}} \Sigma_{\mathrm{X}} \mathrm{V}
$$

where $\Sigma_{\mathrm{X}}=\mathrm{XX}^{\mathrm{T}}$ is the covariance matrix, $\mathrm{V}$ is the eigenvector matrix of $\Sigma_{\mathrm{X}}$ and $\Lambda$ is the corresponding diagonal matrix of eigenvalues. 
As PCA has the property of packing the greatest energy into the least number of principal components, in PCA the eigenvectors corresponding to the $\mathrm{k}$ largest eigenvalues are selected to form a lower dimensional subspace. It is proven that the residual reconstruction error generated by dismissing the $\mathrm{N}_{\mathrm{T}}-\mathrm{k}$ components are low even for small $\mathrm{k}[9]$.

\subsection{Discrete Cosine Transform (DCT)}

The DCT transforms spatial information to decoupled frequency information in the form of DCT coefficients. Also it exhibits excellent energy compaction. The definition of DCT for an $\mathrm{N} \times \mathrm{N}$ image is [10]:

$$
\operatorname{DCT}_{\mathrm{uv}}=\frac{1}{\mathrm{~N}^{2}} \sum_{\mathrm{x}=0}^{\mathrm{N}-1} \sum_{\mathrm{y}=0}^{\mathrm{N}-1} \mathrm{f}(\mathrm{x}, \mathrm{y}) \cos \left[\frac{(2 \mathrm{x}+1) \mathrm{u} \pi}{2 \mathrm{~N}}\right] \cos \left[\frac{(2 \mathrm{y}+1) \mathrm{v} \pi}{2 \mathrm{~N}}\right]
$$

where $f(x, y)$ is $N \times N$ image pixels.

\section{Classifier Design}

Radial Basis Function (RBF) neural networks have found to be very attractive for many engineering problem because: (1) they are universal approximators, (2) they have a very compact topology and (3) their learning speed is very fast because of their locally tuned neurons. Therefore RBF neural networks serve as an excellent candidate for pattern applications and attempt to make the learning process in this type of classification faster than normally required for the multi-layer feed forward neural networks [11-12]. The construction of the RBF neural network involves three different layers with feed forward architecture. The input layer of this network is fully connected to the hidden layer. Connections between the input and hidden layers have unit weights and, as a result, do not have to be trained. The hidden units are also fully connected to the output layer. The goal of the hidden units is to cluster the data and reduces its dimensionality with a nonlinear transformation and maps the input data to a new space. Therefore the transformation from the input space to the hidden space is nonlinear, whereas the transformation from the hidden space to the output space is linear.

The RBF neural network is a class of neural networks, where the activation function of the hidden units is determined by the distance between the input vector and a prototype vector. The activation function of the hidden units is expressed as [11-12]:

$$
\mathrm{R}_{\mathrm{i}}(\mathrm{x})=\mathrm{R}_{\mathrm{i}}\left(\frac{\left\|\mathrm{x}-\mathrm{c}_{\mathrm{i}}\right\|}{\sigma_{\mathrm{i}}}\right) \quad, \quad \mathrm{i}=1,2, \ldots, \mathrm{r}
$$

Where $\mathrm{x}$ is an $\mathrm{n}$-dimensional input feature vector, $\mathrm{c}_{\mathrm{i}}$ is a $\mathrm{n}$-dimensional vector called the center of the hidden unit, $\sigma_{i}$ is the width of hidden unit and $r$ is the number of the hidden units. Typically the activation function of the hidden units is chosen as a Gaussian function with mean vector $c_{i}$ and variance vector $\sigma_{i}$ as follows: 


$$
\mathrm{R}_{\mathrm{i}}(\mathrm{x})=\exp \left(-\frac{\left\|\mathrm{x}-\mathrm{c}_{\mathrm{i}}\right\|^{2}}{\sigma_{\mathrm{i}}^{2}}\right)
$$

Note that $\sigma_{i}^{2}$ represents the diagonal entries of covariance matrix of Gaussian function. The output units are linear and therefore the response of the $\mathrm{j}$-th output unit for input $\mathrm{x}$ is given as:

$$
y_{j}(x)=b(j)+\sum_{i=1}^{r} R_{i}(x) w_{2}(i, j)
$$

Where $w_{2}(i, j)$ is the connection weight of the $i$-th hidden unit to the $j$-th output node and $b(j)$ is the bias of the $j$-th output. The bias is omitted in this network in order to reduce network complexity. Therefore:

$$
y_{j}(x)=\sum_{i=1}^{r} R_{i}(x) \times w_{2}(i, j)
$$

Training RBF neural network can be made faster than the methods used to train multi-layer neural networks. In this paper a Hybrid Learning Algorithm (HLA) based on [12] was used to estimate the width and center of hidden units and synaptic weights.

\section{Experimental Results}

To check the suitability of each feature domain experimental studies are carried out on the ORL database images of Cambridge University. 400 face images from 40 individuals in different states from the ORL database have been used to evaluate the performance of the each feature domain. A total of 200 images have been used to train and another 200 for test. Each training set consists of 5 randomly chosen images from the same individual in the training stage. In the first step after subimage creation, the classifier is trained separately based on each feature domains using HLA technique [12]. In the second step, recognition performance is evaluated. Each test image is projected onto the feature extraction and sent to the classifier. This procedure was repeated for each feature domain. Also in each feature domain the number of feature elements used to represent feature vectors was varied.

The results of the experiments are summarized in Fig. (4) to (7). Fig. (4) shows the average error rate for each feature domains computed for the 40 runs as a function of the number of feature elements. The average error rate curves show that for the PCA a minimum error rate of $1.5 \%$ with 60 elements feature vector is obtainable. Also Fig. (4) shows that the DCT with 40 elements feature vector can yield 3.9\% error rate. It is also clear from this figure the PZM with the proposed feature extraction outperforms the PCA and DCT by providing a minimum error rate of $0.3 \%$ with 21 elements feature vector which is derived from the moments of order 9 and 10. It is interesting to note that although the feature elements of PZM are far less than the other two feature domains, the recognition rate is far superior. 


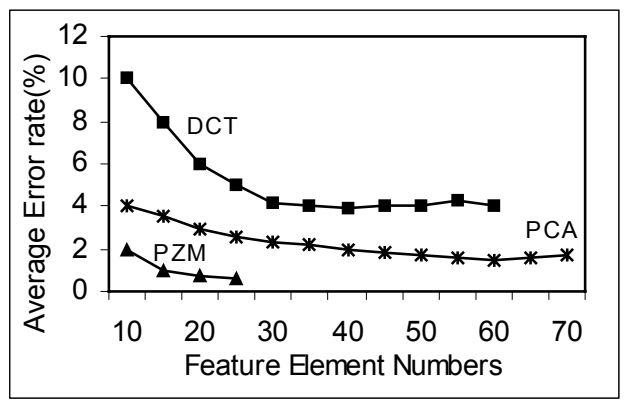

Fig. 4. Average error rate based on feature elements

Another important result revealed by the experiments is shown in Fig. (5). These curves present the standard deviation of the error rate computed for 40 runs for each feature domains. These graphs indicate sensitivity of results to the choice of the training and testing sets. The higher order of the PZM through the proposed feature extraction presents again the lowest standard deviation.

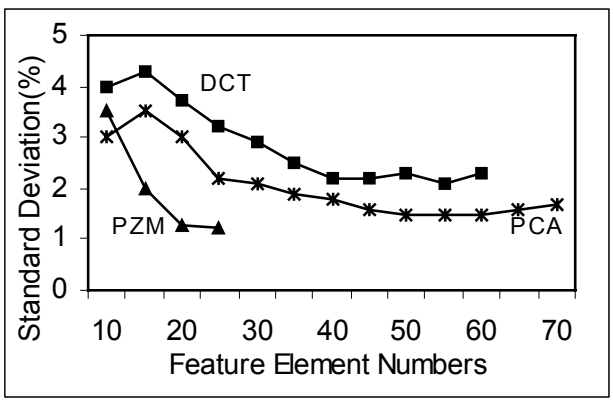

Fig. 5. Standard deviation based on feature elements

As a complement to Fig. (5), the minimum performance among 40 runs as a function of the number of feature elements is plotted in Fig. (6). The PZM presents again the best performance in the experiments. These results show that higher orders of PZM contain more and useful information for face recognition process.

It should be noted that when the PCA was applied to the entire facial image as reported in [13] an error rate of 3\% with the same database was obtained. For the purpose of evaluating how the non-face portion of a face image such as hair, neck, shoulder and background will influence the recognition results we have chosen the PZM with 21 elements, the PCA with 60 elements and the DCT with 40 elements for feature extraction. We have also used RBF neural network with HLA learning algorithm as classifier. We varied the CIR value and evaluated the recognition rate. Fig. (7) shows the effect of CIR on the error rate. As Fig. (7) shows by variation of the CIR value error rate also changes. By defining and using CIR parameter we have obtained a better recognition rate which is $99.3 \%$ for PZM, $98.5 \%$ for PCA and $96.1 \%$ for DCT. The above results were obtained for $\mathrm{CIR}=0.87$ that it is the optimum value for CIR. 


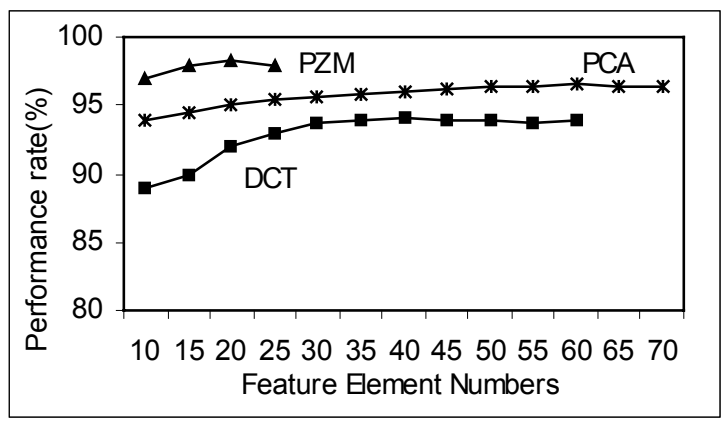

Fig. 6. Minimum performance rate for each feature domain

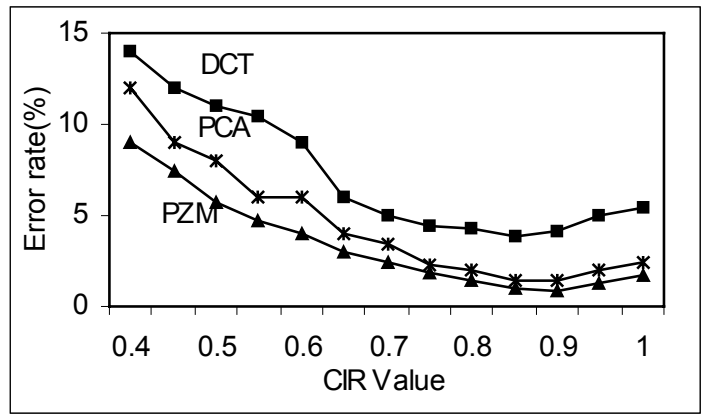

Fig. 7. Error rate based on CIR value

\section{Conclusion}

This paper presented a feature extraction method for the recognition of human faces in 2-Dimensional digital images. The proposed technique utilizes a modified feature extraction technique, which is based on a flexible face localization algorithm followed by various feature domains. This paper has compared several feature domains for human face recognition. These include PZM, PCA and DCT. Also we have introduced CIR parameter for efficient and robust feature extraction technique. It was shown through experimentation the effect of varying this parameter in recognition rate. We have also indicated the optimum value for the CIR for the best recognition results through exhaustive experimentation. We have shown that high order PZM contains very useful information about the facial images. The highest recognition rate of $99.3 \%$ with ORL database was obtained using this proposed algorithm. 


\section{References}

1. Grudin, M. A.: On Internal Representation in face Recognition Systems. Pattern Recognition. Vol. 33, No. 7 (2000) 1161-1177

2. Yung M. H., Kreigman D. J. and Ahuja N., Detecting Face in Images: A Survay, IEEE Trans. on Patt. Anal. and Mach. Intel., Vol. 34, No. 1 (2002) 34-58

3. Haddadnia J., Faez K.: Human Face Recognition Based on Shape Information and Pseudo Zernike Moment. $5^{\text {th }}$ Int. Fall Workshop Vision, Modeling and Visualization, Saarbrucken, Germany, Nov. 22-24 (2000) 113-118

4. Daugman, J.: Face Detection: A Survey. Computer Vision and Image Understanding, Vol. 83, No. 3, Sept. (2001) 236-274

5. Chen L. F., Liao H. M., Lin J. and Han C.: Why Recognition in a statistic-based Face Recognition System should be based on the pure Face Portion: A Probabilistic decision-based Proof. Pattern Recognition, Vol. 34, No. 7 (2001) 1393-1403

6. Zhou W.: Verification of the nonparametric characteristics of backporpagation neural networks for image classification. IEEE Transaction On Geoscience and Remote Sensing, Vol. 37, No. 2, March (1999) 771-779

7. Haddadnia J., Faez K., Moallem P.: Neural Network Based Face Recognition with Moments Invariant. IEEE International Conference On Image Processing, Vol. I, Thessaloniki, Greece, 7-10 October (2001) 1018-1021

8. The C. H. and Chin R. T.: On Image Analysis by the Methods of Moments. IEEE Transaction On Pattern Analysis And Machine Intelligence, Vol. 10, No. 4, (1988) 496-513

9. Truk M. and Pentland A.: Eigenfaces for Recognition. Journal Cognitive Neuroscience, Vol. 3, No. 1 (1991) 71-86

10. Embree P. M. and Kimble B.: C language Algorithm for Digital Signal Processing. Printice Hall, New Jercy, 1991

11. Haddadnia J. and Faez K.: Human Face Recognition Using Radial Basis Function Neural Network. 3rd Int. Conf. On Human and Computer, Aizu, Japan, Sep. 6-9 (2000) 137-142

12. Haddadnia J., Ahmadi M. and Faez K., A Hybrid Learning RBF Neural Network for Human Face Recognition with Pseudo Zernike Moment Invariant, IEEE International Joint conference on Neural Network, Honolulu, HI, May 12-17 (2002), Accepted for presentation

13. Thomaz C. E., Feitosa R. Q. and Veiga A.: Design of Radial Basis Function Network as Classifier in Face Recognition Using Eigenfaces. IEEE Proceedings of Vth Brazilian Symposium on Neural Network, (1998) 118-123 